\title{
Mortality Associated with the Use of Inappropriate Drugs According Beers Criteria: a Systematic Review
}

\author{
Karina Sichieri $^{1}$, Adriano R.Baldacin Rodrigues ${ }^{2}$, Juliana.A.Takahashi ${ }^{3}$, Silvia Regina Secoli ${ }^{4}$, Moacyr \\ Roberto Cuce Nobre ${ }^{5}$, Martinez Altava Mónica ${ }^{6}$, FernándezGarrido Julio ${ }^{7, *}$ \\ ${ }^{1}$ University Hospital, University of São Paulo \\ ${ }^{2}$ Nurse, Heart Institute, Hospital Clinic ofthe Faculty of Medicine, University of São Paulo \\ 3Librarian of the School of Nursing, University of São Paulo \\ ${ }^{4}$ AssociateProfessor of Medical-Surgical Department, School of Nursing, University of São Paulo \\ ${ }^{5}$ Teacherand researcher associated with the Clinical Epidemiology Unit of the Heart Institute, \\ Hosp. Cl. Of the Faculty of Medicine, University of Sao Paulo \\ ${ }^{6}$ Research Nurse, University General Hospital of Castellon (Spain) \\ ${ }^{7}$ Faculty of Nursing and Podiatry, University of Valencia (Spain) \\ *Corresponding Author: julio.fernandez@uv.es
}

Copyright $@ 2013$ Horizon Research Publishing All rights reserved.

\begin{abstract}
The aims of this systematic review are to identify and analyse the scientist literature available evidence about the use of potentially inappropriate medications, according to the Beers Criteria, that is associated with mortality in the elderly people.It have been made a search of publications in most traditional electronic databases among the scientific community (Pubmed / Medline, EMBASE and Web of Science) and it have been selected publications that obey the criteria of 'observational study', 'elderly' and 'Beers Criteria' and that they had as a result the mortality of the study population. After publications selection it proceeded to dump data by two researchers independently to avoid selection bias. The methodological quality of the selected studies was assessed by the checklist Newcastle-Ottawa. The final sample of this systematic review has been made up of 17 studies published in Pubmed and Embase databases majority, 8 of which make up the meta-analysis. In descriptive synthesis has been observed that most of the studies have a level of evidence IV (94'1\%) with cohort delineation (94'1\%) and non-probability sampling technique (70'6\%).Data collection was prospective in $5818 \%$ of cases, with a sample (n) greater than 1000 elderly $(64 \cdot 7 \%)$ and followed up for 6 to 12 months $\left(52^{\prime} 9 \%\right)$.The meta-analysis involving 90.611 elders informed that users who take inappropriate drug according to the Beers Criteria had a higher relative risk for mortality outcome (RR $=1.11,95 \%$ CI 1'01-1'22 P = 0'023), regardless of study stage, comorbidity presence, polypharmacy or type of inappropriate medication used.
\end{abstract}

Keywords Elderly, Inappropriate Medication, Mortality, Systematic Review, Meta-Analysis, Beers Criteria

\section{Introduction}

The current demographic changes, generally characterized by a dramatic improvement in life expectancy and resulting increase in population rates aging is, among other things, showing new health needs, among which include high frequency of comorbidities and using associated polypharmacy. The multiple disorders and their treatments can become triggers for adverse effects associated with potentially inappropriate prescribing drugs that often involves clinical complications and adverse effects on health and disease control.

The use of polypharmacy in elderly, along with aging metabolic changes, has been generating some controversy and doubts about the suitability use of some drugs while on the impact of these drugs, potentially inappropriate, may have on mortality among elderly population. However, despite clinical suspicion, there seems no sufficient scientific evidence to claim that potentially inappropriate medication use (IMU) in the elderly is associated with mortality, so that posed a literature analysis to try to find these evidence. As a reference tool of inappropriate medications it has worked with the Beers criteria, which includes 41 drugs or classes of drugs considered inadequate to use with elderly in any situation and 7 considered unsuitable according to circumstances or doses, both groups also classified according to severity, high or low.

\section{Materials and Methods}

It conducted a systematic review of published studies in electronic databases that showed a positive relationship between inappropriate drug prescription in the elderly and mortality outcome, in order to identify and analyse evidence on the association of use potentially inappropriate drug in the elderly according Beers (IMU) and mortality.

It have been used the Beers criteria because is the most 
widely used internationally to assess the drug therapy adequacy in the elderly.

It were included in the analysis all observational studies conducted with people over 65 years old (in developed countries) or 60 (in developing countries), regardless of the study context (hospital, outpatient or home) and that would make reference to the drugs misuse as the Beers criteria (1991, 1997 and 2003) resulting in death.

It was established as the analysis period, studies published between 1991 (first publication year of Beers criteria) and 2010.

It were excluded from RS, the study sample of frail elderly, because it not determine whether this circumstance was significantly determined outcome of mortality, and also those that show intermediate results, such as RAMs, falls, hospitalizations or other, that somehow could have a decisive influence as a mortality cause.

The databases searched were: Bank CAPES thesis, CINAHL, Current contents connect, Embase, International Lilacs, Pharmaceutical Abstracts, Proquest dissertation and Theses, Pubmed / Medline, Scopus, Web of Science and Science direct.

The initial search provided a total of 1193 articles, their methodological rigor was analysed by Newcastle-Ottawa checklist.After removing duplicate articles or those considered with insufficient scientific rigor, it leaved a sample of 1042 items $(n=1042)$ they were analysed by title and abstract by two researchers independently.Each overturned different data items to a database consisting of following variables: number of study; publishing-related variables (database, type of publication, country, language, authors and area of operation), study purpose, method-related variables (study design, duration, data collection, sample type, stage version of the Beers criteria, outcome information source and age), characteristics of the sample (mean age, sex, level of comorbidities, chronic diseases number, diagnosis, activities assessment of daily living, cognitive assessment, number of drug or class of drugs used), IMU (incidence, number of people who took at least one IMU and its characteristics-gender, age, comorbidities, number of medications-) and results (number of people who died and made use of IMU, measure of effect, confidence intervals, $p$ value and author's conclusion) and evidence level too.

In 12 of the cases were sent an email to the study authors to complete the information specified.It expanded information in 3 cases, but the remaining 9, 3 authors had no more data and 6 did not respond.

In meta-analysis conducting it adopted a random effects model inclusive for subgroup analysis because all studies had different characteristics:different ages of elderly and health conditions, evaluation of IMU by the Beers criteria in 3 different versions and healthcare scenarios differentiated.These different characteristics collected in the analysed studies led to diversity and heterogeneity existence, so the random effect values the contribution of small studies giving results in a wider confidence interval.

In this RS it's considered the heterogeneity test.

Synthesis measurement were taken effect relative risk and the required number of users-cases in which IMU used gave a mortality result ( $\mathrm{NNH}$ ) used to measure complement intervention impact of IMU use.

To check the strength of the results of the meta-analysis was performed a subgroup analysis considering outcome studied (mortality), IMU evaluation by the Beers criteria 2003 version; as a backdrop, the hospital $(n=8)$.

The meta-analysis was performed with L.Bax MIX 2.0 Professional software for meta-analysis in Excel version (2.01.0) Biostat XL 2010 program.

By having an outcome so defined (mortality), it is use controlleddescriptors and keywords as specified search strategy seeking to obtain more relevant studies.However, in the preliminary phase, found no systematic review databases RS (Bandolier, Clinical Evidence, Cochrane Library and other).This study systematic review databases were located in Pubmed / Medline and EMBASE, mostly.

Table No. 1 shows controlled and uncontrolled descriptors for each study used in search, according to PICO* strategy.

Time limit was established as early 1991, because it was the year that Beers criteria arises.As a final limit it was established in 2010. 
Table 1. Controlled and uncontrolled descriptors used in the search, according to PICO strategy in this SR

\begin{tabular}{|c|c|c|}
\hline \multirow[t]{2}{*}{$\mathbf{P}$} & MESH descriptor (Pubmed/Medline) & Aged/ "aged, 80 and over" \\
\hline & Uncontrolled descriptor & centenarian (s) / nonagenarian (s) / octogenarian (s) / senior (s) / geriatric / elder (s) / older / old / ancient \\
\hline \multirow[t]{2}{*}{$\mathbf{I}$} & MESH descriptor & “inappropriate prescribing" \\
\hline & Uncontrolled descriptor & $\begin{array}{l}\text { "inappropriate prescription (s)"/ / suboptimal prescribing" / "inappropriate medication (s)" / "inappropriate drug (s)" / } \\
\text { "inappropriate medicine (s)" / "inadequate prescribing" / "inadequate prescription (s)" / "suboptimal prescription (s)"/ } \\
\text { "suboptimal therapy" / "inadequate medications" / "drug related problem (s)" / "medication-related problem (s)" / beers } \\
\text { / "beers criteria" / "beers list" }\end{array}$ \\
\hline \multirow[t]{2}{*}{$\mathbf{O}$} & MESH descriptor & $\begin{array}{l}\text { Mortality / "mortality [subheading]" / "hospital mortality" / death } \\
\text { / "outcome assessment (health care)" }\end{array}$ \\
\hline & Uncontrolled descriptor & "adverse drug event (s) / "adverse event (s)" / outcome (s) / mortalities / deaths / "adverse medication events" \\
\hline \multirow[t]{2}{*}{$\mathbf{P}$} & DECS descriptor (LILACS)* & Idoso \\
\hline & Uncontrolled descriptor & I \\
\hline \multirow[t]{2}{*}{ I } & DECS descriptor & $\begin{array}{l}\text { "Prescriçãoinadequada"/ "uso de medicamento"/ } \\
\text { "preparaçosfarmacêuticas" }\end{array}$ \\
\hline & Uncontrolled descriptor & "medicamentopotencialmenteinapropriado" \\
\hline \multirow[t]{2}{*}{$\mathbf{P}$} & EMTREE descriptor (EMBASE) & Aged / "aged hospital patient" / "very elderly" / "geriatric patient" \\
\hline & Uncontrolled descriptor & centenarian (s) / nonagenarian (s) / octogenarian (s) / elder (s)/ older / old \\
\hline \multirow[t]{2}{*}{ I } & EMTREE descriptor & "inappropriate prescribing" / prescription / drug / "drug therapy" \\
\hline & Uncontrolled descriptor & $\begin{array}{l}\text { "suboptimal prescribing" / "inappropriate medication (s)" / "inappropriate drug (s)" / "inappropriate medicine (s)" / } \\
\text { "suboptimal prescription (s)" / "suboptimal medication"/ } \\
\text { "suboptimal drug" / "inadequate medication (s)" / "inadequate prescribing" / "inadequate prescription (s)" / } \\
\text { "inadequate drug (s)" }\end{array}$ \\
\hline \multirow[t]{2}{*}{$\mathbf{o}$} & EMTREE descriptor & mortality / death / "adverse outcome" / "drug fatality" \\
\hline & Uncontrolled descriptor & "adverse drug event (s)" \\
\hline \multirow[t]{2}{*}{$\mathbf{P}$} & CINAHL descriptor & aged / "aged, 80 and over" / "aged hospitalized" \\
\hline & Uncontrolled descriptor & centenarian (s) / nonagenarian (s) / octogenarian (s) / senior (s)/ geriatric / elder (s) / older / old / ancient / elderly \\
\hline \multirow[t]{2}{*}{ I } & CINAHL descriptor & drugs / "prescribing patterns" / "drug therapy" / "prescription, drug" / drug utilization" \\
\hline & Uncontrolled descriptor & medication (s) / medicine (s) / prescribing / prescription (s) / inappropriate / inadequate / suboptimal \\
\hline \multirow[t]{2}{*}{$\mathbf{O}$} & CINAHL descriptor & mortality / "hospital mortality" / death / "outcome assessment" / "outcome (health care)" \\
\hline & Uncontrolled descriptor & outcome (s) / "adverse event (s)" / mortalities / deaths / "adverse drug event (s) / "adverse medication event (s)" \\
\hline
\end{tabular}

*Lilacs: portuguesedatabase ;P:patient/population I:intervention/indicator $\quad$ C: comparison/control O: outcome 


\section{Results}

The results are grouped by the following items: study selection process, publications characterization (general, methodological approach) sample characteristics, IMU description, elders groups who used or not IMU and the respective measures effect on the outcome, meta-analyses and subgroup analysesIMU classified by the Beers criteria (2003) and mortality in the hospital setting.

Of the eleven electronic databases consulted in the first stage of study, most studies identifying occurred in Science Direct $(\mathrm{n}=244,20.5 \%)$ and Web of Science $(\mathrm{n}=215,18.0 \%)$.

Of the studies reviewed holistically, the $27.5 \%$ were found in the Pubmed / Medline database $(n=25)$, the $24.2 \%$ in Embase $(\mathrm{n}=23)$ and $23.4 \%$ in IPA $(\mathrm{n}=15)$.

Of the initially selected studies $(\mathrm{n}=1193)$ were excluded $151(12.7 \%)$ because they were duplicates and $1042(87.3 \%)$ remaining 1002 were further excluded after reading the abstract, thus leaving $40(3.8 \%)$ studies who underwent a comprehensive reading.

Comprehensive reading revealed that 25 jobs $(2.4 \%)$ were on non-relevant aspects to the review, which $15(1.4 \%)$ were finally suitable for the sample, but when reviewing the references of these 15 studies, was decided to analyse and include two more articles in the final sample being selected a total of $n=17$.

The following Table 2 shows the articles general characteristics included in this SR:

Regarding the methodological approach, we found that most of the studies had a level of evidence IV $(n=16 ; 94.1 \%)$ with cohortdelineation $(n=16 ; 94.1 \%)$, non-probability sampling technique $(n=12 ; 70.6 \%)$, data collected prospectively $(n=10$, $58.8 \%)$, larger sample of 1000 elderly $(\mathrm{n}=11 ; 64.7 \%)$ with followingof $12-24$ months $(\mathrm{n}=9,52.9 \%)$ and mortality results obtained by database query $(\mathrm{n}=13 ; 76.5 \%)$.

Regarding the characteristics of the sample, showed that the majority of studies involved mainly female individuals $(\mathrm{n}=16$; $94.1 \%)$ with an average age greater than 70 years $(\mathrm{n}=14,82.4 \%)$ and using at least two drugs $(\mathrm{n}=15 ; 88.2 \%)$.

In 9 of the 17 studies $(52.9 \%)$, the researchers described the number of comorbidities using the Charlson comorbidity index $(\mathrm{n}=5 ; 29$ '4\%).

Others $9\left(52^{\prime} 9 \%\right)$ also described the elderly functional assessment byscales Barthel, Katz and others.

The mean number of drugs used in the elderly was higher in those studies examining medicines use in long-standing institutions $\left(\mathrm{E} 1^{1}, \mathrm{E} 3^{6}, \mathrm{E} 9^{4}, \mathrm{E} 14^{21}\right.$, and in Table 2) than in the hospital setting $\left(\mathrm{E} 4^{24}, \mathrm{E} 6^{18}, \mathrm{E} 12^{19}, \mathrm{E} 16^{5}\right.$, in Table 2$)$ and this, more than in the community $\left(\mathrm{E} 1^{1}, \mathrm{E} 2^{2}, \mathrm{E} 5^{23}, \mathrm{E} 10^{8}, \mathrm{E} 13^{13}, \mathrm{E} 15^{11}\right.$, in Table 2$)$.

C: community; LSI: long-standing institution, ND: data not described in the study

In most studies $(\mathrm{n}=12 ; 70.5 \%)$ IMU was classified as aninappropriate drug independently of diagnosis, therapy dose or time.In $23.5 \%$ of cases $\left(\mathrm{E}^{6}, \mathrm{E} 6^{18}, \mathrm{E} 11^{14}, \mathrm{E} 16^{5}\right)$ it's given a more extended classification, taking into account the drug-pathology interaction condition. Of these four studies, only 2 reported the results.

Table 2. General characteristics of the publications included in the SR

\begin{tabular}{|c|c|c|c|c|c|c|}
\hline Studio & Authors & Title & $\begin{array}{l}\text { Journal/yea } \\
\mathrm{r}\end{array}$ & $\begin{array}{l}\text { Publication } \\
\text { language } \\
\text { (country of } \\
\text { publication) }\end{array}$ & $\begin{array}{l}\text { Knowledge } \\
\text { area }\end{array}$ & Database \\
\hline E01 $^{1}$ & $\begin{array}{l}\text { Barnett K, } \\
\text { McCowan C, } \\
\text { Evans JMM, } \\
\text { Gillespie ND, } \\
\text { Davey PG, Fahey } \\
\text { T }\end{array}$ & $\begin{array}{l}\text { Prevalence and outcomes of use } \\
\text { of potentially inappropriate } \\
\text { medicines in older people: } \\
\text { Cohort study stratified by } \\
\text { residence in nursing home or in } \\
\text { the community }\end{array}$ & $\begin{array}{l}\text { BMJ } \\
\text { Quality and } \\
\text { Safety/ } \\
2011\end{array}$ & English (UK) & $\begin{array}{l}\text { Public } \\
\text { Health }\end{array}$ & $\begin{array}{l}\text { Embase; } \\
\text { Web of } \\
\text { science; } \\
\text { Pubmed; } \\
\text { CINAHL }\end{array}$ \\
\hline $\mathbf{E 0 2}^{2}$ & $\begin{array}{l}\text { Beer C, Hyde Z, } \\
\text { Almeida } \\
\text { OP, Norman P, } \\
\text { Hankey GJ, } \\
\text { Yeap BB, Flicker } \\
\text { L }\end{array}$ & $\begin{array}{l}\text { Quality use of medicines and } \\
\text { health outcomes among a } \\
\text { cohort of community dwelling } \\
\text { older men: an observational } \\
\text { study }\end{array}$ & $\begin{array}{l}\text { British } \\
\text { Journal of } \\
\text { Clinical } \\
\text { Pharmacolo } \\
\text { gy/2011 }\end{array}$ & $\begin{array}{l}\text { English } \\
\text { (AUST) }\end{array}$ & Medicine & Pubmed \\
\hline $\mathbf{E 0 3}^{6}$ & $\begin{array}{l}\text { Dedhiya SD, } \\
\text { Hancock E, } \\
\text { Craig BA, } \\
\text { Doebbeling CC, } \\
\text { Thomas J }\end{array}$ & $\begin{array}{l}\text { Incident Use and Outcomes } \\
\text { Associated With Potentially } \\
\text { Inappropriate Medication Use } \\
\text { in Older Adults }\end{array}$ & $\begin{array}{l}\text { The } \\
\text { American } \\
\text { Journal } \\
\text { of Geriatric } \\
\text { Pharmacoth } \\
\text { erapy/2010 }\end{array}$ & English (USA) & Pharmacy & $\begin{array}{l}\text { Pubmed; } \\
\text { Embase; } \\
\text { Scopus; } \\
\text { Web of } \\
\text { Science }\end{array}$ \\
\hline E04 $4^{25}$ & Szlejf C & $\begin{array}{l}\text { Eventos adversos médicos em } \\
\text { idosos hospitalizados:frequência } \\
\text { e fatores de riscoemenfermaria } \\
\text { de geriatria }\end{array}$ & $\begin{array}{l}\mathrm{PhD} \\
\text { thesis/2010 }\end{array}$ & $\begin{array}{l}\text { Portuguese } \\
\text { (BRA) }\end{array}$ & Medicine & CAPES \\
\hline
\end{tabular}




\begin{tabular}{|c|c|c|}
\hline $\mathbf{E}^{23}$ & $\begin{array}{l}\text { Pozzi C, Lapi F, } \\
\text { Mazzaglia, G, } \\
\text { Inzitari M, } \\
\text { Boncinelli } \\
\text { M,Geppetti P, } \\
\text { MugelliA,Marchi } \\
\text { onni N, Bari M }\end{array}$ & $\begin{array}{l}\text { Is suboptimal prescribing arisk } \\
\text { factor for poor healthoutcomes } \\
\text { in community welling elders? } \\
\text { The ICAReDicomano study }\end{array}$ \\
\hline E06 $^{18}$ & $\begin{array}{l}\text { Mansur N, Weiss } \\
\text { A, } \\
\text { Beloosesy Y }\end{array}$ & $\begin{array}{l}\text { Is there an associationbetween } \\
\text { inappropriateprescription drug } \\
\text { use andadherence in } \\
\text { dischargedelderly patients? }\end{array}$ \\
\hline$E 07^{15}$ & $\begin{array}{l}\text { Lin H, Liao C-C, } \\
\text { Cheng S-H, } \\
\text { Wang P-W, } \\
\text { Hsueh Y-S }\end{array}$ & $\begin{array}{l}\text { Association of potentially } \\
\text { inappropriate medication use } \\
\text { with adverse outcomes } \\
\text { inambulatory elderly } \\
\text { patientswith chronic diseases - } \\
\text { Experience in a } \\
\text { TaiwaneseMedical Setting }\end{array}$ \\
\hline
\end{tabular}

$\mathbf{E 0 8}^{\mathbf{2 1}}$ Page RL, Ruscin The risk of adverse drugevents $\mathrm{JM}$

$\begin{array}{ll}\text { Raivio MM, } & \text { Use of Inappropriate } \\ \text { Laurila JV, } & \text { Medications } \\ \text { Strandberg TE, } & \text { and Their } \\ \text { Tilvis RS, } & \text { PrognosticSignificance Among } \\ \text { Pitkälä KH } & \text { In-Hospitaland Nursing Home } \\ & \text { Patients withand without } \\ & \text { Dementia in Finland }\end{array}$

$\mathbf{E 1 0}^{\mathbf{8}}$ Espino DV, BazalduaOV,Pal mer RF, Mouton CP,Parchman ML, Miles TP,Markides K.

E11 $^{14}$ Lau DT, Kasper JD, Potter DEB, Lyles A, Bennett RG

E12 $^{19}$ Onder G, Landi F, Liperoti R, Fialova D, Gambassi G, Bernabei R

$\mathbf{E 1 3}^{13}$ Klarin I, Wimo A, Fastbom J and hospital-relatedmorbidity and mortality amongolder adults with potentiallyinappropriate medication use

The

American

Journal

of Geriatric

Pharmacoth

erapy/2006

Drugs

Aging/2006

Suboptimal medication useand mortality in an oldercommunity based cohort:results from the Hispanic EPESE Study

Hospitalization and deathassociated with potentiallyinappropriate medicationprescriptions among elderlynursing home residents

Impact of inappropriate druguse among hospitalized olderadults

European

Journal of

Clinical

Pharmacolo gy/2005

The association ofinappropriate drug use withhospitalisation and mortality -

A population-based study ofthe very old

gerontol.

Series

A,Biologic

al sciences

andmedical

sciences $/ 20$

06

Arquives of

Internal

Medicine/2

005

$\begin{array}{lll}\text { English } & \text { Medicine } & \text { Pubmed; } \\ \text { (ITALY) } & & \text { Embase; } \\ & & \text { Web of } \\ & \text { Science }\end{array}$

$\begin{array}{lll}\text { English } & \text { Medicine } & \text { Pubmed; } \\ \text { (ISRAEL) } & & \text { Embase; } \\ & & \text { IPA; } \\ & \text { Scopus; } \\ & \text { Web of } \\ & & \text { Science } \\ \text { English } & \text { Pharmacy } & \text { Pubmed; } \\ \text { (TAIWAN) } & & \text { Scopus; } \\ & \text { Embase; } \\ & \text { IPA; Web } \\ & \text { of } \\ & \text { science; } \\ & \text { CINAHL; } \\ & & \text { Current } \\ & & \text { Contents } \\ & & \text { Connect } \\ \text { English(USA) } & \text { Pharmace } \\ & & \text { Sirect; } \\ & & \text { Scopus; } \\ & & \text { Pubmed }\end{array}$

English

Medicine

Embase;

Current

Contents

Connect;

Web of

Science;

IPA;

CINAHL

References

English (USA) Medicine

Pubmed;W

ebofScienc

e;IPA;

Embase; $\mathrm{Cu}$

rrentConten

tsConnect;

Scopus

English Medicine

(ITALY)

Pubmed;W

ebofScienc

e;CurrentC

ontentsCon

nect;Scopu

Drugs English (USA) Public Pubmed;

Aging/2005 Health Scopus;

Embase;

IPA; Web

of

science;

CINAHL;

Current

Contents

Connect 


\begin{tabular}{|c|c|c|c|c|c|c|}
\hline E14 ${ }^{21}$ & $\begin{array}{l}\text { Perri III M, } \\
\text { Menon } \\
\text { AM,Deshpande } \\
\text { AD, } \\
\text { ShindeSB,Jiang } \\
\text { R, Cooper JW, } \\
\text { CookCL, Griffin } \\
\text { SC, Lorys RA }\end{array}$ & $\begin{array}{l}\text { Adverse outcomes } \\
\text { associatedwith inappropriate } \\
\text { drug use innursing homes }\end{array}$ & $\begin{array}{l}\text { The Annals } \\
\text { of } \\
\text { Pharmacoth } \\
\text { erapy/2005 }\end{array}$ & English (USA) & Pharmacy & $\begin{array}{l}\text { Pubmed; } \\
\text { Embase; } \\
\text { Web of } \\
\text { Science; } \\
\text { IPA; } \\
\text { Scopus }\end{array}$ \\
\hline $\mathbf{E}^{15^{11}}$ & $\begin{array}{l}\text { Hanlon JT, } \\
\text { FillenbaumGG,K } \\
\text { uchibhatla M, } \\
\text { ArtzMB,Boult C, } \\
\text { Gross CR, } \\
\text { Garrard } \\
\text { J. ,Schmader KE }\end{array}$ & $\begin{array}{l}\text { Impact of inappropriate druguse } \\
\text { on mortality and functionalstatus } \\
\text { in representativecommunity } \\
\text { dwelling elders }\end{array}$ & $\begin{array}{l}\text { Medical } \\
\text { care/2002 }\end{array}$ & English (USA) & Pharmacy & $\begin{array}{l}\text { Scopus; } \\
\text { Web of } \\
\text { Science; } \\
\text { CINAHL; } \\
\text { Pubmed }\end{array}$ \\
\hline E16 $^{5}$ & $\begin{array}{l}\text { Chin MH, Wang } \\
\text { LC, Jin } \\
\text { L,Mulliken R, } \\
\text { Walter J, } \\
\text { HayleyDC, } \\
\text { Karrison TG, } \\
\text { Nerney } \\
\text { MP, Miller A, } \\
\text { Friedmann PD }\end{array}$ & $\begin{array}{l}\text { Appropriateness of medication } \\
\text { selection for older persons inan } \\
\text { urban academicemergency } \\
\text { department }\end{array}$ & $\begin{array}{l}\text { Academic } \\
\text { Emergency } \\
\text { Medicine/1 } \\
999\end{array}$ & English (USA) & Medicine & $\begin{array}{l}\text { Pubmed; } \\
\text { Embase; } \\
\text { Web of } \\
\text { Science; } \\
\text { Scopus }\end{array}$ \\
\hline E1 $7^{10}$ & $\begin{array}{l}\text { Gupta S, } \\
\text { Rappaport HM, } \\
\text { Bennett LT }\end{array}$ & $\begin{array}{l}\text { Inappropriate DrugPrescribing } \\
\text { and RelatedOutcomes for } \\
\text { Elderly } \\
\text { Medicaid BeneficiariesResiding } \\
\text { in Nursing Homes }\end{array}$ & $\begin{array}{l}\text { Clinical } \\
\text { Therapeutic } \\
\text { s/1996 }\end{array}$ & English (USA) & Pharmacy & References \\
\hline
\end{tabular}

Table 3. Features based on the sample of seniors in the studies included in the SR.

\begin{tabular}{|c|c|c|c|c|c|c|c|}
\hline Studio & Sex & $\begin{array}{l}\text { Age } \\
\text { (mean in } \\
\text { years) }\end{array}$ & Diagnosis & $\begin{array}{l}\text { number of } \\
\text { chronic } \\
\text { diseases( } \\
\text { mean)/ } \\
\text { scale of } \\
\text { comorbidi } \\
\text { ties } \\
\text { (mean) }\end{array}$ & functional evaluation & $\begin{array}{l}\text { cognitive } \\
\text { evaluatio } \\
\mathrm{n}\end{array}$ & $\begin{array}{l}\text { number of drugs } \\
\text { / class of drugs } \\
\text { used mean }\end{array}$ \\
\hline E1 $^{1}$ & $\begin{array}{l}\text { C: } \\
57^{\prime} 0 \% \\
\text { female } \\
\text { LSI: } \\
72{ }^{\prime} 3 \% \\
\text { female }\end{array}$ & $\begin{array}{l}66 \text { to } 99 \\
\text { (C: } 75 \text { '2 } \\
\text { LSI: } \\
84^{\prime} 5 \text { ) }\end{array}$ & ND & ND & ND & $\mathrm{ND}$ & $\begin{array}{l}\text { C: mean - } 8,8 \\
\text { classes / elderly } \\
\text { LSI: 11'6 mean } \\
\text { class / elderly }\end{array}$ \\
\hline $\mathbf{E 2}^{2}$ & $\begin{array}{l}100 \% \\
\text { male }\end{array}$ & $\begin{array}{l}65 \text { to } 83 \\
(77)\end{array}$ & ND & $\begin{array}{l}\text { Charlson } \\
1-2 \text { points } \\
(19,2 \%)\end{array}$ & ND & $\mathrm{ND}$ & $\begin{array}{l}\geq 5 \\
\text { drugs: } 35,8 \% \\
\text { elderly }\end{array}$ \\
\hline $\mathbf{E 3}^{6}$ & $\begin{array}{l}76{ }^{\prime} 5 \% \\
\text { female }\end{array}$ & $\begin{array}{l}\geq 65 \\
\left(83{ }^{\prime} 1\right)\end{array}$ & ND & $\begin{array}{l}\text { Charlson: } \\
\text { 2'25 }\end{array}$ & ND & $\mathrm{ND}$ & $\begin{array}{l}1 \text { to } 10: 24{ }^{\prime} 3 \% \\
\text { elderly } \\
11 \text { to } 15: 28^{\prime} 3 \% \\
\text { elderly } \\
16 \text { to } 20: 23^{\prime} 4 \% \\
\text { elderly } \\
+20: 24^{\prime} 0 \% \\
\text { elderly }\end{array}$ \\
\hline $\mathbf{E} 4^{25}$ & $\begin{array}{l}59^{\prime} 1 \% \\
\text { female }\end{array}$ & $\begin{array}{l}\geq 60 \\
\left(78^{\prime} 1\right)\end{array}$ & $\begin{array}{l}\text { Depression }\left(18^{\prime} 7 \%\right) \\
\text { Dementia }\left(29^{\prime} 8 \%\right) \\
\text { Delirium }\left(35^{\prime} 7 \%\right) \\
\text { Infection }\left(55^{\prime} 6 \%\right)\end{array}$ & $\begin{array}{l}\text { Charlson: } \\
\text { 3’07 }\end{array}$ & $\begin{array}{l}\text { Katz index independent } \\
\text { Mean:4'13 }\end{array}$ & $\mathrm{ND}$ & $\begin{array}{l}\text { Mean: 5’73 } \\
\text { drugs }\end{array}$ \\
\hline $\mathbf{E 5}^{23}$ & $\begin{array}{l}57 ' 1 \% \\
\text { female }\end{array}$ & $\begin{array}{l}\geq 65 \\
\left(73 \pm 6^{\prime} 8\right)\end{array}$ & ND & $\begin{array}{l}2 ’ 3 \\
\text { diseases }\end{array}$ & $\begin{array}{l}\text { Disability in basic } \\
\text { activity }=7,5 \% \\
\text { Disability in } \\
\text { instrumental activity = } \\
32 \%\end{array}$ & $\mathrm{ND}$ & $\begin{array}{l}\text { Mean: 2’2 } \\
\text { drugs/elderly } \\
\geq \text { 5drugs: } 12{ }^{\prime} 3 \%\end{array}$ \\
\hline $\mathbf{E}^{18}$ & $61 \% \%$ & $\geq 65$ & Neurological (18\%) & $6 ’ 3$ & Katz index & Mini-me & $5^{\prime} 9$ at discharge \\
\hline
\end{tabular}




\begin{tabular}{|c|c|c|c|c|c|c|c|}
\hline & female & $\left(81^{\prime} 1\right)$ & $\begin{array}{l}\text { Orthopaedic (18\%) } \\
\text { Fall and imbalance } \\
(15 \%) \\
\text { Hemato-oncology } \\
(11 \%)\end{array}$ & diseases & $\begin{array}{l}\text { Independent (58\%) } \\
\text { Partial }\left(27^{\prime} 4 \%\right) \\
\text { Total }\left(14^{\prime} 6 \%\right)\end{array}$ & $\begin{array}{l}\text { ntal: } \\
\text { Normal } \\
\left(78^{\prime} 8 \%\right) \\
\text { Mild to } \\
\text { moderate } \\
\left(12^{\prime} 7 \%\right) \\
\text { Severe } \\
\left(8^{\prime} 5 \%\right)\end{array}$ & $\begin{array}{l}6 ' 5 \text { after a month } \\
\text { of discharge }\end{array}$ \\
\hline $\mathbf{E} 7^{15}$ & $\begin{array}{l}55^{\prime} 5 \% \\
\text { female }\end{array}$ & $\begin{array}{l}\geq 65 \\
\left(744^{\prime} 7\right)\end{array}$ & $\begin{array}{l}\text { Hypertension } \\
\left(24^{\prime} 2 \%\right) \\
\text { Heart disease } \\
\left(14^{\prime} 1 \%\right) \\
\text { Diabetes mellitus } \\
\left(11^{\prime} 5 \%\right)\end{array}$ & $\begin{array}{l}1 \text { disease } \\
(27,8 \%) \\
2 \text { diseases } \\
(46 \%) \\
3 \text { diseases } \\
\left(211^{\prime} \%\right) \\
\geq 4 \\
\text { diseases } \\
(5 \%)\end{array}$ & ND & $\mathrm{ND}$ & $\begin{array}{l}\leq 4 \text { drugs } \\
\left(800^{\prime} 8 \%\right)\end{array}$ \\
\hline $\mathbf{E 8}^{21}$ & $\begin{array}{l}68^{\prime} 9 \% \\
\text { female }\end{array}$ & $\begin{array}{l}\geq 75 \\
(79)\end{array}$ & ND & ND & ND & ND & ND \\
\hline $\mathbf{E 9}^{24}$ & $\begin{array}{l}82^{\prime} 0 \% \\
\text { female }\end{array}$ & $\begin{array}{l}\geq 70 \\
(86)\end{array}$ & ND & $\begin{array}{l}\text { Charlsons } \\
\text { cale } \\
\text { Dementia } \\
\text { :> } 2 \text { points } \\
\left(42^{\prime} 4 \%\right) \\
\text { Without } \\
\text { dementia: } \\
>2 \text { points } \\
\left(30^{\prime} 6 \%\right)\end{array}$ & $\begin{array}{l}\text { Clinical Dementia } \\
\text { Rating scale } \\
\text { Dependence among } \\
\text { elderly with dementia: } \\
76^{\prime} 9 \% \\
\text { Dependence among } \\
\text { older adults without } \\
\text { dementia: } 21 ' 2 \%\end{array}$ & $\begin{array}{l}\text { Mini-me } \\
\text { ntal } \\
\text { Dementia } \\
\text { 9'9 } \\
\text { points } \\
\text { (mean) }\end{array}$ & Mean: 9'0 drugs \\
\hline $\mathbf{E 1 0}^{8}$ & $\begin{array}{l}64{ }^{\prime} 5 \% \\
\text { female }\end{array}$ & $\begin{array}{l}65 \text { to } 99 \\
(73)\end{array}$ & $\begin{array}{l}\text { Cardiovascular } \\
\text { disease }(12 ' 5 \%) \\
\text { Neoplasia }\left(6^{\prime} 3 \%\right) \\
\text { Diabetes mellitus } \\
\left(32^{\prime} 9 \%\right) \\
\text { Hypertension } \\
\left(56^{\prime} 9 \%\right)\end{array}$ & ND & $\begin{array}{l}\text { Older American } \\
\text { Resource } \\
\text { Scale } \\
\text { Disability in basic } \\
\text { activity }=14 \text { '5\% } \\
\text { Disability in } \\
\text { instrumental activity = } \\
41 ' 5 \%\end{array}$ & $\mathrm{ND}$ & $\begin{array}{l}>4 \\
\text { Drugs }=21{ }^{\prime} 1 \%\end{array}$ \\
\hline E11 $^{14}$ & $\begin{array}{l}73^{\prime} 8 \% \\
\text { female }\end{array}$ & $\geq 65$ & Dementia (47’7\%) & ND & $\begin{array}{l}0-3 \text { limitations }\left(26^{\prime} 3 \%\right) \\
4-5 \text { limitations }\left(30^{\prime} 3 \%\right) \\
6 \text { limitations }\left(46^{\prime} 2 \%\right)\end{array}$ & ND & ND \\
\hline E12 $^{19}$ & $\begin{array}{l}52{ }^{\prime} 2 \% \\
\text { female }\end{array}$ & $\begin{array}{l}\geq 65 \\
\left(78^{\prime} 8\right)\end{array}$ & $\begin{array}{l}\text { Hypertension } \\
\left(37^{\prime} 8 \%\right) \\
\text { Ischemic heart } \\
\text { disease }\left(27^{\prime} 6 \%\right) \\
\text { Heart failure } \\
\left(21^{\prime} 5 \%\right) \\
\text { Diabetes }\left(20{ }^{\prime} 2 \%\right)\end{array}$ & $\begin{array}{l}\text { Charlson } \\
\geq 2 \text { points } \\
\left(47^{\prime} 8 \%\right)\end{array}$ & $\begin{array}{l}\text { Disability in basic } \\
\text { activity }=38,5 \%\end{array}$ & $\begin{array}{l}\text { Cognitiv } \\
\mathrm{e} \\
\text { impairme } \\
\mathrm{nt} \\
\left(34^{\prime} 6 \%\right)\end{array}$ & $\begin{array}{l}-\geq 5 \\
\text { drugs: } 74{ }^{\prime} 3 \%\end{array}$ \\
\hline $\mathbf{E}^{13^{13}}$ & $\begin{array}{l}57^{\prime} 9 \% \\
\text { female }\end{array}$ & $\begin{array}{l}\geq 75 \\
\left(82^{\prime} 6\right)\end{array}$ & ND & ND & ND & ND & $\begin{array}{l}\text { Mean: } 4^{\prime} 4 \text { drugs } \\
\geq 5 \text { drugs: } 40^{\prime} 1 \%\end{array}$ \\
\hline $\mathbf{E} 14^{21}$ & $\begin{array}{l}81^{\prime} 6 \% \\
\text { female }\end{array}$ & $\begin{array}{l}\geq 65 \\
\left(84^{\prime} 6\right)\end{array}$ & $\begin{array}{l}\text { Dementia } \\
\left(70^{\prime} 5 \%\right) \\
\text { Heart disease } \\
\left(66^{\prime} 9 \%\right) \\
\text { Hypertension } \\
\left(57^{\prime} 1 \%\right)\end{array}$ & ND & ND & ND & $\begin{array}{l}\text { Mean: } 8 \text { '7 drugs } \\
\geq 5 \text { drugs: } 76^{\prime} 5 \%\end{array}$ \\
\hline $\mathbf{E}^{15^{11}}$ & $\begin{array}{l}64{ }^{\prime} 8 \% \\
\text { female }\end{array}$ & $\geq 65$ & ND & ND & $\begin{array}{l}\text { Katz Index, } \\
\text { Older } \\
\text { American Resource } \\
\text { Scale and } \\
\text { Rosow-Breslau Scale } \\
\text { Disability in one or } \\
\text { more basic activity: } \\
18^{\prime} 3 \% \\
\text { Disability in } \\
\text { instrumental activity = } \\
35^{\prime} 5 \%\end{array}$ & $\begin{array}{l}\text { Cognitiv } \\
\text { e } \\
\text { impairme } \\
\text { nt } \\
(15,8 \%)\end{array}$ & $\begin{array}{l}2 \text { to } 4 \text { drugs: } \\
42^{\prime} 9 \%\end{array}$ \\
\hline E16 $6^{5}$ & $\begin{array}{l}63{ }^{\prime} 0 \% \\
\text { female }\end{array}$ & $\begin{array}{l}\geq 65 \\
\left(76^{\prime} 3\right)\end{array}$ & ND & $\begin{array}{l}\text { 1'8 } \\
\text { diseases }\end{array}$ & $\begin{array}{l}\text { Inability to, at least, one } \\
\text { basic activity: } 57 \%\end{array}$ & ND & $\begin{array}{l}\text { Mean: } 3^{\prime} 6 \\
\text { Drugs: } 73^{\prime} 6 \%\end{array}$ \\
\hline $\mathbf{E} 17^{10}$ & $73^{\prime} 6 \%$ & $60 ' 1 \%-$ & ND & ND & $\mathrm{ND}$ & $\mathrm{ND}$ & ND \\
\hline
\end{tabular}




\begin{aligned} & \hline female $\geq 81 \\ &$ C: community ND: Not described $\quad$ LSI: long standing institution \\ & \hline\end{aligned}

In the overall analysis following IMU were important:

Table 4. Drug groups most commonly found

\begin{tabular}{ccc}
\hline DRUG GROUP & $\begin{array}{c}\text { Gravity according side effects } \\
\text { probability }\end{array}$ & $\mathrm{n}$ \\
\hline Anticholinergic-Antihistamines & High & 9 \\
Benzodiazepines & High & 10 \\
Tricyclicantidepressants & High & 8 \\
Antiplatelet & Low & 6 \\
Musclerelaxants & High & 8 \\
Antispasmodics & High & 6 \\
Digoxin & Low & 6 \\
Ferroussulphate & Low & \\
\hline
\end{tabular}

Whichever version of Beers and studied scenario is agreement between the different studies that benzodiazepine (BDZ) are the drugs most identified as IMU.The long-acting BDZ should be avoided entirely in this population because of its effect of prolonged sedation, risk of falls and consequent fractures. Secondly, tricyclic antidepressants, antihistamines and anticholinergic also achieve high frequency as IMU and should be avoided due to their sedative and central nervous system depressants.

Reviewing Beers 2012 includes this recommendation cited and classified as evidence IV and recommendation grade D.

The $47.1 \%$ studies used Beers version $2003\left(\mathrm{E} 1^{1}, \mathrm{E} 2^{2}, \mathrm{E} 3^{6}, \mathrm{E} 4^{25}, \mathrm{E} 6^{18}, \mathrm{E} 7^{15}, \mathrm{E} 8^{21}, \mathrm{E} 12^{19}\right.$, and in Table 2).

The measure of studies effect was the Odds ratio $(\mathrm{n}=7 ; 41.2 \%)$.

In most of the studies ( $\mathrm{n}=11 ; 64.7 \%)$ authors observed no association between the use of IMU and mortality.Only in 3 of them $\left(E 3^{6}, E 11^{14}, E 14^{21}\right.$, in Table 2$)$ it was showed this association.

Were included in the meta-analysis 8 items $\left(E 1^{1}, E 3^{6}, E 4^{25}, E 5^{23}, E 7^{15}, E 8^{21}, E 9^{24}, E 12^{19}\right.$ in Table 2) with a total of 90611 participants aged.Here there was a statistically significant difference between elderly groups $(\mathrm{p}=0.022)$ regardless to mortality and a large heterogeneity of the studies $\left(\mathrm{I}^{2}=53.86 \%\right)$.

Regarding the subgroup analysis, it was performed with 6 studies using Beers version 2003, with a total of 89164 users verifying anstatistical difference among elderly users of IMU for mortality risk $(\mathrm{p}=0.021)$ and with great heterogeneity $\left(\mathrm{I}^{2}=62.09 \%\right)$.

Subgroup analysis on the 3 studies conducted in hospitals $\left(\mathrm{E} 4^{25}, \mathrm{E} 6^{18}, \mathrm{E} 12^{19}, \mathrm{n}=5530\right.$ elderly) demonstrated no statistical difference groups with mortality as a result $(\mathrm{p}=0.246)$ and moderate heterogeneity $\left(\mathrm{I}^{2}=28.91 \%\right)$.

Table 5 shows the synthesis of meta-analysis results including subgroup analysis.

Table 5. Summary of results of meta-analyses, including subgroup analyses.

\begin{tabular}{|c|c|c|c|c|c|c|c|c|}
\hline Outcome & $\begin{array}{c}\text { Number of } \\
\text { studies }\end{array}$ & $\begin{array}{c}\text { Total } \\
\text { patients }\end{array}$ & $\mathrm{Q}(\mathrm{p})$ & $\begin{array}{c}\mathrm{I} 2 \\
\text { (IC 95\%) }\end{array}$ & $\begin{array}{c}\text { overall } \\
\text { estimate } \\
\text { (IC 95\%) }\end{array}$ & $\mathrm{p}$ & $\mathrm{NNH}$ & Conclusion \\
\hline $\begin{array}{l}\text { Mortality } \\
\text { from all } \\
\text { causes }\end{array}$ & 8 & 90611 & $\begin{array}{c}15^{\prime} 17 \\
\left(0^{\prime} 033\right)\end{array}$ & $\begin{array}{c}53^{\prime} 86^{\circ} \%(0 \mathrm{a} \\
79)\end{array}$ & $\begin{array}{c}111\left(1^{\prime} 01 \mathrm{a}\right. \\
\left.1{ }^{\prime} 22\right)\end{array}$ & $0 ’ 022$ & 36 & $\begin{array}{l}\text { Statistical } \\
\text { difference }\end{array}$ \\
\hline $\begin{array}{c}\text { Mortality } \\
\text { versus Beers } \\
2003 \\
\text { standard }\end{array}$ & 6 & 89164 & $13 ’ 18$ & $\begin{array}{l}62,09 \% \\
(7 \text { a } 84)\end{array}$ & $\begin{array}{c}1,12 \\
(1,02 \text { a } 1,25)\end{array}$ & 0’021 & 33 & $\begin{array}{l}\text { Statistical } \\
\text { difference }\end{array}$ \\
\hline $\begin{array}{l}\text { Mortality in } \\
\text { the hospital }\end{array}$ & 3 & 5.530 & $\begin{array}{c}2,81 \\
(0,244)\end{array}$ & $\begin{array}{l}28,91 \% \\
(0 \text { a } 92)\end{array}$ & $\begin{array}{c}0,81 \\
(0,57 \text { a } 1,15)\end{array}$ & $0 ’ 246$ & 151 & $\begin{array}{c}\text { No statistical } \\
\text { difference }\end{array}$ \\
\hline
\end{tabular}




\section{Discussion}

This SR has found evidence to suggest that the elderly who used IMU had higher relative risk of death $(\mathrm{RR}=1.11$, 95\% CI 1.01-1.22, $\mathrm{p}=0.023$ ), according Beers criteria, regardless of the stage of study, comorbidities, polypharmacy or type of IMU used.

In descriptive synthesis were included $1.6 \%$ of the total studies reviewed by title and abstract, results were quite similar to other RS that analysed the use of IMU $(2 \%, 3 \%)^{7}$ 9,20 .

This SR also found as general common characteristics to other studies the close relationship between aging and criteria development to assess the quality of care.Generally, these studies were conducted in developed countries, being USA the first to develop a criterion for classifying IMU.

The study has been carried out predominantly from cohort studies, predominantly those of nature population and hospital environment held in long-stay institutions, with prospective data collection and non-probability sampling.Although it no represents the highest level of evidence, Phase IV studies whose data are obtained from databases are particularly relevant because the information has been obtained from actual conditions (heterogeneous population from various care settings and representative actual health services).

The profile of the sample of elderly, found that female sex is prevalent in all studies of the SR, except one (E2 ${ }^{2}$, in Table 2).This can be explained because older women tend to live longer than men, regardless of the stage and health conditions, older women are more likely to use IMU.

This association of women and IMU may be due to biological matters (most exposed to health problems), psychological (worry more about their health / symptoms) and socio-cultural (most frequently used health services).

In previous SR also showed that female gender is a risk factor associated with the use of $\mathrm{IMU}^{3,4,16,17}$

In all studies, age adopted the "elders" was in $>65$ years, according to the WHO, except Brazil, which defined over 60 years old as a person aged.

In reference to the characteristics of the sample were found to age, number of diseases and medicines as well as the setting of care are variables that must be considered together because of their close relationship, because the older, larger number of diseases and drugs to treat.In addition there is usually an increase in hospitalizations number, and this population, are the ultimate users of long-stay facilities (nursing homes).

The analysis reveals that the average age $>80$ years and the average number of drugs consumed were higher for institutionalized elderly. These are generally more vulnerable to IMU use as other groups of elderly due to frailty and comorbidity present.Also showed that is a risk associated with the use of IMU in long-stay institutions have more than 85 years and comorbidity ( $>3$ Charlson index).

Moreover, the existence of multiple physicians prescribing carries an increased risk of polypharmacy, which in turn is set as a contributing factor to use of IMU.This is especially common in long-stay institutions which is corroborated by the evidence that SR has shown that the use of IMU is more pronounced among institutionalized elderly that in other healthcare settings.

The fact that in some studies the authors did not clearly define the IMU concept (in terms of dose or therapy time) can be confusing, but always has been associated with older people.

In the meta-analysis were included 8 studies reporting results with absolute frequency on sample and the results analysed, mortality.For this result was statistically significant difference $(p=0.02,95 \%$ CI $1.01-1.22)$ between the two groups showing the harmful effects of the use of IMU for elderly as mortality outcome.This relationship, against the non-confirmation on the previous SR and refuted in other studies of the SR, is also found in three studies $\left(\mathrm{E}^{6}{ }^{6}\right.$, $\mathrm{E} 11^{14}, \mathrm{E} 14^{21}$, in Table 2) who obtained higher risk of death for the elderly in the IMU use.

The studies heterogeneity was large $\left(\mathrm{I}^{2}=53.86 \%\right)$ due to the differences in study scenarios (long-stay institutions (LSI), hospital and community, and community LSI), comorbidities users profile, number and class of drugs used as well as differences between drugs among countries.

In the subgroup analysis $(\mathrm{n}=5530)$ in hospital setting, there was less heterogeneity of the studies $\left(\mathrm{I}^{2}=28.91 \%\right)$ because users came from hospitals and drugs were classified according to 2003 Beers.

The meta-analysis, provides no statistically significant difference between the groups, but showing a trend of lower mortality risk for elderly IMU users. This could be explained because in the hospital environment more patients are supervised by professionals on adverse drug effects and prescribing precautions IMU.However, more studies are needed to reinforce or refute these hypotheses given the weakness of the data.

The use of random effect in this SR is justified by the studies diversity and heterogeneity.This model values small studies contribution providing a broader IC.

$\mathrm{NNH}$ calculation for mortality from all causes was 36 , i.e., for every 36 seniors who used IMU, one would die.In subgroup analysis yielded a similar result, $\mathrm{NHH}=33$. By contrast, in subgroup analysis carried out with hospital studies was $\mathrm{NHH}=151$, which, in accordance with meta-analysis interpretation, we infer that the use of IMU in hospital setting protects the elderly of mortality outcome since each 151 IMU user, one perish.

Drug therapy responds to the balance between the benefit and the harm a drug can bring.That is, the drug is prescribed looking for a positive effect of the same in order to try to improve individuals' health because generally, without the drug, there's not an improvement in health, sometimes even more damage.The problem arises in these patients with multiple disorders and visits to different specialists, each one prescribing in their health branch, may guide to incompatible 
pharmacology situations and side effects, usually negative (morbidity and mortality).Thus the use of drugs requires an integrated interdisciplinary management and with the help of tool in health posed by the Beers criteria, Professionals can carry it out.The fact that an IMU-mortality relationship has been established, although not clearly evidenced yet, should encourage practitioners to treat pharmacologically with care on users and find strong results about it.

\section{Limitations}

This RS found limited to information availability from primary studies.The absence of responses to requests made to the authors of primary studies did not enable the inclusion of several studies in the meta-analysis, having been able to increase the sample to strengthen, with the possibility of showing a different result.

Moreover, comorbidity, different drugs, and the type of facility or research context are a limitation by itself because heterogeneity that each of these variables can result.

\section{REFERENCES}

[1] Barnett K, McCowan C, Evans JMM, Gillespie ND, Davey PG, Fahey T.Prevalence and outcomes of Potentially Inappropriate use of medicines in older people: Cohort study stratified by residence in nursing home or in the community.BMJ QualSaf.2011, 20 (3) :275-81.

[2] Beer C, Hyde Z, Almeida OP, Norman P,HankeyGJ, Yeap $\mathrm{BB}$, et al.Quality use of medicines and health outcomes Among a cohort of community dwelling older men: an observational study.Br JClinPharmacol.2011, 71 (4) :592-99.

[3] Bierman AS, Pugh MJV, Dhalla I, Amuan M, Fincke G, Rosen A, et al.Sex differences in prescribing Inappropriate Among elderly veterans.Am

GeriatrPharmacother.2007;5:147-161.

[4] Buck MD, Atreia A, Brunker CP, Jain A, Suh TT, Palmer RM, et al.Potentially Inappropriate medication prescribing in outpatientspractices: Prevalence and Characteristics based on electronic patient health records. Am J GeriatrPharmacother.2009;7 (2) :84-92.

[5] Chin MH, Wang LC, Jin L, Mulliken R, Walter J, Hayley DC, et al.Appropriateness of medication selection for older persons in an urban academic emergency department. AcadEmerg Med1999;6 (12) :1232-42.

[6] Dedhiya SD, Hancock E, Craig BA, Doebbeling CC, Thomas J.Incident use and outcomes Associated with Potentially Inappropriate medication use in older adults.AmJGeriatrPharmacother. 2010, 8 (6) :562-70.

[7] Dimitrow MS, Airaksinen MSA, Kivela SL, Lyles A, LeikolaSNS.Comparison of prescribing criteria to evaluate-appropriateness of drug treatment in Individuals aged 65 and older: a systematic review.JAGS. 2011;59:1521-30.
[8] Espino DV, OV Bazaldua, Palmer RF, Mouton CP, Parchman ML, Miles TP, MarkidesK.Suboptimal medication use and mortality in an older community based cohort: results from the Hispanic EPESE Study.JGerontol A BiolSci Med Sci 2006;61 (2) :170-5.

[9] Guaraldo L, Cano FG, GS Damasceno, RozenfeldS.Inappropriate medication use Among the elderly: a systematic review of administrative databases.BMC Geriatrics.2011;11:79.

[10] Gupta S, Rappaport HM, Bennett LT.Inappropriate Drug Prescribing and Related Outcomes for Elderly Medicaid Beneficiaries Residing in Nursing Homes. ClinTher.1996, 18 (1) : 183-96.

[11] Hanlon JT, Fillenbaum GG, Kuchibhatla M, Artz MB, Boult $\mathrm{C}$, Gross $\mathrm{CR}$, et al.Impact of Inappropriate drug use on mortality and functional status in representative community dwelling elders.Care Med.2002, 40 (2) :166-76.

[12] Janus E, AparasuR.Healthcare Associated outcomes with beers' Criteria: a systematic review.Geriatrics. 2007;41:438-48.

[13] Klarin I, Wimo A, Fastbom J. The association of inappropriate drug use with hospitalization and mortality. A population-based study of thevery old. Drugs Aging. 2005, 22(1): 69-82.

[14] Lau DT, Kasper JD, Potter DEB, Lyles A, Bennet RG.Hospitalization and Death Associated with Potentially Inappropriate medication prescriptions Among elderly nursing home residents.Arch Intern Med 2005, 165:68-74.

[15] Lin H, Liao CC, Cheng SH, Wang PW,HsuehYS.Association of Potentially Inappropriate Use With medication adverse outcomes in ambulatory elderly patients with chronic diseases -experience in a Taiwanese medical setting.Drugs Aging.2008, 25 (1) :49-59.

[16] Locatelli J, Lira AR, Torraga LKLA, AT Paes. Inappropriate medicationusing the Beers criteria in Brazilian elderly hospitalized patients.ConsultPharm.2010;25:36-40.

[17] Maio V, Del Canale S, Abouzaid S.GAP Investigators.Usingexplicitcriteria to evaluate-the quality of prescribing in elderly Italianoutpatients: a cohort study.J ClinPharmTher.2010;35:219 - 229.

[18] Mansur N, Weiss A, Beloosesky Y.Is there an association Between Inappropriate prescription drug use and adherence in discharged elderly patients?Ann Pharmacother.2009, 43 (2) :177-84.

[19] Onder G, Landi F, Liperoti R, Fialova D, Gambassi G, BernabeiR.Impact of Inappropriate drug Use among hospitalized older adults. EurJ ClinPharmacol.2005, 61:453-9.

[20] OpondoD, Eslami S, Visscher S, de Rooij SE, Verheij R, Korevaar JC, et al.Inappropriateness of medication prescriptions to elderly patients in the primary care setting: a systematic review.PLoSONE.2012, 7 (8): e43617.

[21] Page RL, JM Ruscin. The risk of adverse drug events and hospital related morbidity and mortality Among older adults with Potentially Inappropriate medication use.Am J GeriatrPharmacother2006;4 (4) :297-305.

[22] Perri III M, Menon AM, Deshpande AD, Shinde SB, Jiang R, 
Cooper JW, et al.Associated with Adverse outcomes Inappropriate drug use in nursing homes.Ann Pharmacother.2005, 39 (3) :405-11.

[23] Pozzi C, Lapi F, Mazzaglia G, Inzitari M, Boncinelli M, Geppetti P, et al. Suboptimal prescribing Is a risk factor for poor health outcomes in community-dwelling elders?TheiCareDicomano study.

PharmacoepidemiolDrugSaf.2010, 19 (9) :954-60.
[24] Raivio MM, Laurila JV, Strandberg TE, Tilvis RS, PITK ä 1 äKH. Inappropriate Use of Medications and Their Prognostic Significance Among In-Hospital and Nursing Home Patients with and without Dementia in Finland.Drugs Aging.2006, 23 (4) :333-43.

[25] Szlejf C. Eventos adversos medicosemidosos hospitalizados: frequência e fatores de risco emenfermaria de geriatria [tese]. São Paulo: Faculdade de Medicina, Universidade de São Paulo; 2010 